\title{
Leveillula taurica (Lév) Arn : cultures axéniques, biologie et spécificité parasitaire
}

\author{
PM Molot, JP Leroux, M Diop-Bruckler
}

INRA, Pathologie végétale, 84140 Montfavet, France

(Reçu le 22 décembre 1989; accepté le 11 juin 1990)

\begin{abstract}
Résumé - Leveillula taurica se différencie des autres oïdiums par plusieurs caractéristiques :
- la conservation des isolats s'effectue sur feuilles en survie (et non sur cotylédons) maintenues sur milieu gélosé en atmosphère saturée en eau, la contamination s'effectuant de préférence par la face inférieure du limbe et en présence d'eau. Pour les feuilles de tomate, il convient d'enrichir le milieu avec $\uparrow \mathrm{mg} / \mathrm{l}$ d'acide indol-butyrique;

- la germination des spores s'effectue parfaitement en milieu liquide. Différentes préparations fongicides ont été testées dans ces conditions; aucune ne s'est révélée totalement efficace, même à fortes concentrations;

- grâce à une technique spéciale de microscopie, il est montré que les tubes germinatifs pénètrent par les stomates; cependant ceux-ci ne semblent pas jouer un rôle important dans la sensibilité du matériel végétal;

- sur plantes en serre, les contaminations artificielles peuvent aisément se réaliser avec un inoculum liquide. II est inutile de placer le matériel en chambre humide. Les résultats obtenus en infections croisées montrent qu'il n'y a pas de spécificité parasitaire chez $L$ taurica ( 4 isolats utilisés sur 5 hôtes différents).
\end{abstract}

contamination croisée / "oïdiotron" / milieu de culture de tissus / germination des spores / fongicide / technique microscopique / miniaturisation / hygrométrie / stomate / tomate / concombre / artichaut / cardon

Summary - Leveillula taurica (Lev) Arn: axenic cultures, biology and parasitic specificity. $L$ taurica is distinguished from the other powdery mildews by the following:

- Conservation of isolates is carried out on detached leaves (not on cotyledons) maintained on agar medium (100\% relative humidity) on which the spores are deposited in a drop of water on the lower side of the blade (table I). For young tomato leaves, indole butyric acid $(1 \mathrm{mg} / \mathrm{l})$ should be added to the medium to prevent foliar necrosis (table II).

- The spores germinate very well in liquid medium. Several fungicides have been tested under these conditions: even at high concentrations, none of them have proved to be really efficient (table III).

- A special microscopic technique has shown that the germinative tubes enter via stomata (fig 1). However, they seem to play a minor role in contamination.

For artificial contamination in the greenhouse, the plants are sprayed with liquid inoculum. It is not necessary to place the material in a damp chamber. The results obtained from cross-infections (4 isolates used on 5 host plants) suggest that there is no parasitic specificity (table IV).

The different characteristics noted above are discussed.

cross contamination / "oïdiotron" / tissue culture medium / spore germination / fungicide / microscopic technic / size reduction / humidity / stomata / tomato / cucumber / artichoke / cardoon

\section{INTRODUCTION}

Principalement répandu dans le centre et l'ouest de l'Asie ainsi que dans les régions méditerranéennes et tropicales, Leveillula taurica est susceptible d'attaquer un grand nombre de végétaux (plus de 1000 espèces appartenant à 390 genres regroupés dans 74 familles botaniques). Les plantes spontanées parasitées sont surtout les Composées et les Légumineuses, tandis que les plantes cultivées se rattachent d'abord aux Solanacées (tomate, piment, pomme de terre).

À la différence des autres agents cryptogamiques responsables des oïdiums (Sphaerotheca, Erysiphe, Uncinula, Podosphaera), $L$ taurica se caractérise par un développement mycélien à la fois interne et externe. 
Les conditions de milieu présidant à l'infection et à l'apparition des symptômes diffèrent beaucoup selon l'hôte étudié, mais d'une façon générale la maladie se développe plutôt par temps chaud et sec (sauf pour le piment) et concerne plutôt les feuilles de l'étage moyen de plantes assez âgées ayant atteint le stade de la fructification (Koren, 1978; Palti, 1988; Diop-Bruckler, 1989).

Connu de longue date, le parasite a été étudié en France pour la 1 re fois par Tramier en 1963. $\mathrm{Au}$ cours de la dernière décennie, il a pris une extension croissante (Blancard et Rieuf, 1981), notamment sur cultures de tomate sous abri où la lutte chimique demeure très difficile (Piquemal et al, 1985).

$L$ taurica est un parasite obligatoire dont la culture artificielle n'est possible que sur un support végétal vivant. Des feuilles de piment dont le pétiole est repiqué sur milieu gélosé spécial permettent d'assurer, en conditions axéniques, la conservation des souches pendant environ 2 mois (Molot et al, 1987a et b). Cependant cette technique n'est pas toujours aisée - l'utilisation de bocaux pose des problèmes d'encombrement - et elle ne semble pas, très favorable, par ailleurs, à la réussite des repiquages (volume trop important pour qu'il y ait condensation d'eau sur le feuillage).

Dans la présente étude, nous nous proposons d'aborder à nouveau le problème de la conservation en cherchant, d'une part à miniaturiser la technique, et d'autre part à l'adapter au maintien en survie de feuilles d'autres espèces que le piment. En choisissant de travailler en boîtes de Petri plutôt qu'en bocaux, nous créons des conditions de milieu très particulières, différentes de celles recherchées pour les oïdiums classiques; de leur étude devrait découler une meilleure connaissance de la biologie de $L$ tauri$c a$, notamment en ce qui concerne la germina- tion et la pénétration des spores. Enfin, par des contaminations artificielles effectuées sur plantes entières en “oïdiotron" (Fauvel et Molot, 1985), nous contrôlerons le pouvoir pathogène des souches d'origine différente de notre collection et préciserons, par des infections croisées, s'il y a ou non spécificité parasitaire.

\section{MATÉRIEL ET MÉTHODES}

\section{Matériel végétal et cryptogamique}

Nous avons utilisé 4 isolats de $L$ taurica, issus respectivement de cultures en plein champ de piment, tomate, concombre et artichaut. Ce matériel est conservé soit sur ces mêmes plantes, élevées entièrement en enceintes climatiques pressurisées, à l'abri de toute source de contamination (Fauvel et Molot, 1985), soit en culture in vitro sur feuille de piment, selon une technique décrite par ailleurs (Molot et al, $1987 a$ et b) et à laquelle ont été apportées plusieurs modifications (voir plus loin).

Cinq plantes hôtes ont servi aux contaminations artificielles : piment (Yolo Wonder), tomate (Monalbo), concombre (Marketer), artichaut (Jaja) et cardon (Blanc amélioré).

\section{Conservation du matériel fongique}

Si la désinfection du support végétal et des spores du champignon reste identique aux conditions précédemment définies : trempage dans $\mathrm{HgCl}_{2}$ à $0,5 \%$ o pendant 3 min pour les feuilles et 1,5 min pour les oïdies (Molot et al, 1987a), en revanche plusieurs modifications ont été apportées dans le choix des milieux de culture (voir composition en annexe) et dans la façon de "repiquer" le champignon, celui-ci étant apporté de préférence dans une goutte d'eau sur la face inférieure du limbe.

Tableau I. Développement de $L$ taurica sur feuilles en survie de 3 plantes hôtes en fonction du mode de contamination. Les résultats sont exprimés en pourcentage par rapport au nombre total de colonies apparues par espèce; pour chaque traitement, 12 feuilles ont été contaminées en 6 points. Comparaison par le test de Duncan au seuil de 0,01.

Inoculation Concombre Piment Tomate

$\begin{array}{llrlrlrl}\text { À sec } & \text { Face supérieure } & 7 & \mathrm{a} & 17 & \mathrm{a} & 8 & \mathrm{a} \\ & \text { Face inférieure } & 33 & \mathrm{c} & 28 & \mathrm{~b} & 31 & \mathrm{c} \\ \text { Par suspension } & \text { Face supérieure } & 16 & \mathrm{~b} & 11 & \mathrm{a} & 15 & \mathrm{~b} \\ \text { dans l'eau } & \text { Face inférieure } & 44 & \mathrm{c} & 44 & \mathrm{c} & 46 & \mathrm{~d}\end{array}$




\section{Germination de spores en milieu liquide}

Contrairement à Erysiphe et Sphaerotheca pour lesquels la germination exige l'absence complète d'eau liquide, les oïdies de $L$ taurica, mises en suspension dans l'eau, émettent sans difficulté 1 ou 2 tubes germinatifs dans les conditions du laboratoire $\left(22^{\circ} \mathrm{C}\right.$, lumière du jour). Nous avons mis à profit cette propriété pour étudier l'action de quelques fongicides antioïdiums classiques : lors de l'emploi, on mélange volume à volume une suspension de spores et une préparation fongicide, l'une et l'autre étant à une concentration double de celle désirée. Les observations se font généralement avec 25000 spores $/ \mathrm{ml}$.

\section{Technique de contamination artificielle}

La contamination des feuilles en survie s'effectue à la surface du limbe en 6 points différents : l'inoculum est apporté à l'aide d'un pinceau, soit à sec, soit dans une goutte d'eau $(50 \mu l)$ préalablement déposée à la surface du limbe. Dans ce dernier cas, la concentration finale en spores est en moyenne de 60 000/ml.

Sur plantes adultes, nous pratiquons toujours les contaminations par pulvérisation d'un inoculum, titrant 60000 spores $/ \mathrm{ml}$. L'adjonction de triton $\times 100$ facilite la réussite de l'opération (Molot et Lecoq, 1986). Il est inutile, après la pulvérisation, de mettre les plantes en chambre humide. L'incubation s'effectue en condition d'isolation parfaite, en "oïdiotron" (Fauvel et Molot, 1985) : elle est de l'ordre de 3 semaines à une température moyenne de $25^{\circ} \mathrm{C}$ sous $70 \%$ d'hygrométrie relative moyenne, sans éclairage d'appoint.

\section{Technique d'étude du mode de pénétration du champignon}

À la face inférieure d'une feuille adulte de piment, on dépose en différents endroits une goute d'inoculum $(10 \mu \mathrm{l})$ titrant $40000 \mathrm{spores} / \mathrm{ml}$. Vingt-quatre heure après, la goutte est résorbée. On recouvre alors le point d'inoculation d'une goutte de bleu-coton que l'on sèche aussitôt à l'air chaud; puis on dépose soigneusement à l'aide d'un pinceau un peu de vernis TZ2 (Cyrille France, Paris) afin d'épouser fidèlement les empreintes de l'épiderme; enfin, cette couche de vernis est arrachée à l'aide d'un petit morceau de ruban adhésif et fixée sur lame de microscope pour observation.

\section{RÉSULTATS}

\section{Conservation des souches}

Des essais préliminaires (Molot et al, 1987a) ont montré que l'entretien de $L$ taurica sur feuilles adultes de piment maintenues en survie dans des bocaux était possible. Cependant, la nécessité de travailler de façon moins encombrante nous a conduits à utiliser des boîtes de Petri plutôt que des bocaux, et à choisir par conséquent des organes foliaires de dimension plus réduite : jeunes feuilles ou cotylédons. Cette étude a été menée à la fois sur concombre, piment et tomate.

L'expérimentation effectuée sur jeunes feuilles en survie donne de bons résultats en contaminations artificielles. Celles-ci doivent être pratiquées de préférence à la face inférieure du limbe; de plus, l'apport d'inoculum sous forme d'une suspension de spores dans l'eau se révèle beaucoup plus opérant qu'un apport à sec (tableau I).

Pour accroître la durée de survie de la feuille de concombre, il est cependant souhaitable d'obtenir un bon enracinement. Ce but est atteint en partie en augmentant la quantité de vitamines, notamment celles du groupe $B$, et en enrichissant le milieu en fer (Molot et al, 1987b).

Un problème identique se pose pour la tomate. Il est compliqué en outre par le fait que les limbes, plaqués à la surface du milieu, jaunissent et finissent par nécroser. Parmi les $5 \mathrm{mi}$ lieux expérimentés (tableau II), le milieu renfermant $1 \mathrm{mg} / \mathrm{l}$ l'acide indol-butyrique (AIB) donne les meilleurs résultats.

La conservation de la feuille d'artichaut, même en l'absence de racines, ne présente aucune difficulté majeure.

Signalons enfin que les cotylédons des plantes hôtes concernées ne conviennent pas à la culture de $L$ taurica, leur durée de survie (en-

Tableau II. Pourcentage de folioles de tomate présentant des nécroses du limbe après mise en survie sur différents milieux (voir composition des milieux en annexe). Chaque traitement comporte en moyenne 28 folioles. Comparaison par le test de Duncan au seuil de 0,01 . Milieux L esculentum L hirsutum L peruvianum
(Monalbo)

\begin{tabular}{lrlllll} 
AIB & 9 & a & 10 & a & 14 & a \\
KNOP & 45 & b & 31 & b & 33 & b \\
MSE & 70 & c & 42 & bc & 45 & c \\
Piment & 43 & b & 48 & bc & 51 & c \\
RM & 59 & bc & 50 & bc & 37 & bc \\
T51 & 61 & c & 53 & c & 30 & b \\
\hline
\end{tabular}


viron 15 j) étant nettement inférieure à la période d'incubation du parasite (environ $21 \mathrm{j}$ à $25^{\circ} \mathrm{C}$ ).

\section{Essai de germination de spores en milieu liquide. Application aux tests de produits fongicides (tableau III)}

Comme les contaminations artificielles avec $L$ taurica réussissent bien en atmosphère saturée d'humidité, nous avons eu l'idée d'observer la germination des spores en suspension dans l'eau. Nous avons ainsi constaté qu'elles germaient sans difficulté en moins de $24 \mathrm{~h}$, le tube germinatif, généralement unique, se formant de préférence à l'extrémité inférieure de la spore.

Cette propriété, exceptionnelle pour un oïdium, nous a incités à réaliser des germinations de spores provenant de piment adulte et mises en suspension dans des préparations fongicides. Huit fongicides classiques, couramment utilisés dans la lutte vis-à-vis des Erysiphacées, ont été utilisés : bénomyl, bitertanol, bupirimate, chinométhiomate, fénarimol, soufre, triadiméfon, triforine tous à 2 concentrations, celle préconisée par le fabricant et une autre 4 fois supérieure (sauf pour le bupirimate). On constate, en dépit de différences significatives entre les produits, qu'aucun d'entre eux n'est totalement efficace aux doses conseillées et que le fait de quadrupler la concentration n'apporte pas de réduction importante du pourcentage de germination, notamment en ce qui concerne le chinométhionate et le triadiméfon. Par ailleurs, on observe, en présence de fongicides, une diminution générale de la longueur des tubes germinatifs, mais celleci n'est pas proportionnelle à l'augmentation de matière active.

\section{Mode de pénétration (fig 1)}

La germination des spores en présence d'eau s'effectuant correctement dans des proportions élevées, nous avons cherché à faire des observations directes de germination au contact de l'épiderme de la face inférieure de la feuille de piment, particulièrement riche en stomates.

Environ 3 à $4 \mathrm{~h}$ après le dépôt de la goutte d'inoculum, toutes les conidies viables ont germé. Les tubes germinatifs se développent principalement à partir de l'extrémité inférieure de la spore; ils peuvent passer près d'un stomate ou même par-dessus sans y pénétrer; le long du tube germinatif, on peut observer des appressoriums, mais ceux-ci semblent incapables d'assurer la pénétration directe du champignon à travers la cuticule; la pénétration définitive

Tableau III. Effet de différents fongicides sur la germination des spores de $L$ taurica. Les doses de matière active préconisées par le fabricant contre les oïdiums classiques sont soulignées. Comparaison par le test de Duncan au seuil de 0,01 .

Produits

Concentrations en matière active $\left(m g *^{-1}\right)$
Pourcentages

de spores germées
Moyennes des

longueurs des

tubes germinatifs

(en $\mu \mathrm{m}$ )

$\begin{array}{lr}\text { Témoin eau } & \\ \text { Bénomyl } & 3 \\ & 12 \\ \text { Bitertanol } & \underline{100} \\ & 400 \\ \text { Bupirimate } & \underline{500} \\ \text { Chinométhionate } & 1000 \\ & 7.5 \\ \text { Fénarimol } & 30 \\ & \underline{24} \\ \text { Soufre } & 96 \\ \text { Triadiméfon } & \underline{2475} \\ \text { Triforine } & \underline{0.75} \\ & 1 \frac{285}{140}\end{array}$

$\begin{array}{rll}56 & \mathrm{a} & 102 \\ 8 & \mathrm{c} & 93 \\ 3 & \mathrm{c} & 17,3 \\ 8 & \mathrm{c} & 61 \\ 2 & \mathrm{c} & 46 \\ 13 & \mathrm{bc} & 51 \\ 8 & \mathrm{c} & 45,7 \\ 10 & \mathrm{c} & 84 \\ 8 & \mathrm{c} & 59 \\ 22 & \mathrm{~b} & 58 \\ 7 & \mathrm{c} & 51 \\ 20 & \mathrm{bc} & 1 \\ 9 & \mathrm{c} & 94 \\ 7 & \mathrm{c} & 36,6 \\ 12,5 & \mathrm{bc} & 56 \\ 6 & \mathrm{c} & 49\end{array}$




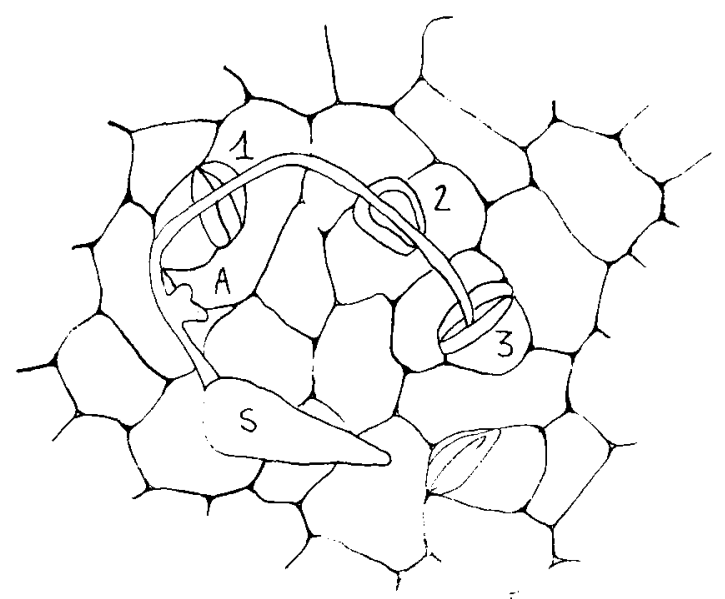

Fig 1. Spore terminale de $L$ taurica (S) germant à la surface inférieure d'une feuille de piment. Après avoir formé un appressorium $(A)$, le tube germinatif passe au-dessus d'un premier (1) puis d'un deuxième stomate (2) avant de pénétrer par l'orifice du troisième (3).

s'effectue par un stomate choisi apparemment au hasard; à ce niveau, on ne remarque pas d'appressorium.

\section{Spécificité parasitaire}

Avec 4 sources différentes d'inoculum prélevées respectivement sur piment, tomate, concombre ou artichaut, nous avons contaminé ces mêmes plantes en y ajoutant le cardon, cette opération étant réalisée par pulvérisation, en conditions isolées («oïdiotron»).

Le tableau IV résume l'ensemble des connaissances acquises à ce jour. II mentionne nos propres travaux (résultats encadrés) et ceux de divers auteurs. L'origine de la souche ne semble pas intervenir dans la réussite des contaminations artificielles et ce, quelle que soit la plante hôte. Nous montrons en particulier que la souche tomate est agressive sur piment, concombre, artichaut et cardon et que la souche artichaut peut faire des dégâts sur cardon.

$L$ taurica semble donc dépourvu de spécificité parasitaire.

\section{DISCUSSION - CONCLUSION}

Les expériences menées dans le cadre de cette étude montrent qu'il est souhaitable de miniaturiser la technique de conservation de $L$ taurica. $\mathrm{Ce}$ faisant, on est conduit à travailler avec de jeunes feuilles de dimension réduite. Dès lors, s'est posé un problème de nécroses foliaires, notamment dans le cas de la tomate, lié en grande partie à la composition du milieu gélosé utilisé. L'adjonction de $1 \mathrm{mg} / \mathrm{l}$ d'acide indolbutyrique améliore beaucoup la situation.

Contrairement aux autres oïdiums (Sphaerotheca, Erysiphe) dont la durée d'incubation à $22{ }^{\circ} \mathrm{C}$ relativement réduite $(5-10$ jours) permet le développement sur cotylédons, $L$ taurica ne peut se conserver sur ce type d'organes qui, après mise en survie, dégénèrent avant l'apparition des fructifications fongiques.

La survie des jeunes feuilles, réalisée en boîte de Petri de faible volume, entraîne la formation d'une abondante condensation favorable aux contaminations artificielles, surtout si elles sont pratiquées à la face inférieure du limbe. La présence d'eau liquide, considérée comme un facteur néfaste pour les oïdiums (Zaracovitis, 1964), se révèle donc bénéfique chez $L$ taurica.

Tableau IV. Contaminations croisées réalisées avec quatre isolats de $L$ taurica. Les résultats nouveaux et originaux sont encadrés; les références bibliographiques mentionnées correspondent à des résultats positifs; le signe ? indique que la contamination n'a pas été réalisée. Chaque contamination comporte un effectif de 3 plantes.

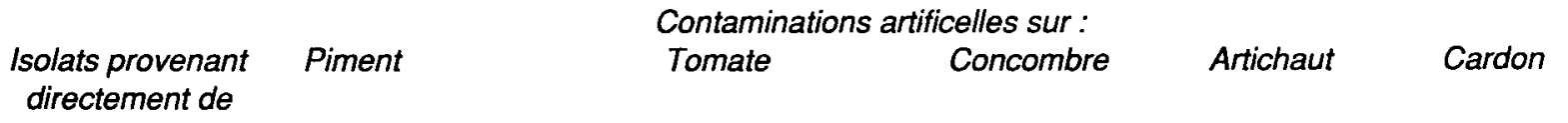

\begin{tabular}{|c|c|c|c|c|c|}
\hline Piment & + & + & Tramier, 1963 & Tramier, 1963 & + \\
\hline Tomate & + & + & + & + & + \\
\hline Concombre & Saad et al, 1972 & $?$ & + & $?$ & $?$ \\
\hline Artichaut & Tramier, 1963 & Besri et Hormattalah, 1985 & $?$ & + & + \\
\hline
\end{tabular}


Cette particularité avait déjà été signalée par Palti en 1971.

Confirmant ces observations, la germination des spores de $L$ taurica a été réalisée in vitro en milieu liquide. Cette caractéristique du parasite permet d'envisager une simplification considérable des tests fongicides, où la recherche de molécules anti-oïdiums fait toujours appel à des dispositifs expérimentaux plus ou moins sophistiqués.

Grâce à une méthode originale simple, nous avons pu suivre le cheminement du tube germinatif à la surface du limbe d'une feuille de piment et sa pénétration par les stomates, confirmant ainsi des travaux japonais (Kunoh et al, 1979; Homma et al, 1980).

En rapprochant cette observation du fait que la contamination s'effectue préférentiellement par la face inférieure du limbe (beaucoup plus riche en stomates que la face supérieure), on pourrait penser que les stomates jouent un rôle déterminant dans l'infection, d'autant plus que les conditions d'hygrométrie élevées, présidant à la réussite des contaminations, favorisent leur ouverture. En fait, il semble qu'il n'en soit rien, car aucune corrélation n'existe entre la richesse en stomates et la sensibilité à $L$ taurica (Pochard, 1982; Daubeze et al, 1989). Si l'infection se fait par les stomates, le déterminisme de la résistance est à rechercher à un autre niveau, peut-être dans les tissus lacuneux ou palissadiques; des études histologiques fines pourraient apporter les éclaircissements souhaités.

Indépendamment de la question des stomates, les facteurs climatiques semblent jouer un rôle capital dans l'expression des symptômes. Si l'influence des températures élevées apparaît généralement propice à la maladie, celle de l'hygrométrie est beaucoup plus discutable, les réponses étant différentes selon les plantes et, pour une même plante, selon les variétés. C'est ainsi que la tomate est plus sensible que le piment en condition sèche et vice-versa (Reuveni et Rotem, 1973) et que, chez le piment, deux génotypes différents (Yolo Wonder et lignées 855) sont l'un sensible en atmosphère sèche et l'autre en atmosphère humide (DiopBruckler, 1989). II convient donc de tenir compte de la très forte interaction génotype-milieu (Pochard, 1982).

La chute prématurée des feuilles, considérée à juste titre comme la conséquence d'une attaque de $L$ taurica, est aussi sous la dépendance du taux d'hygrométrie. Si celui-ci est faible, il y a chute de feuilles chez le piment (alors que l'infection est généralement réduite), tandis que, chez la tomate, les feuilles restent toujours en place (Palti, 1988).

La question de la spécificité parasitaire de $L$ taurica revêt un grand intérêt pratique. Pour tenter de la résoudre, nous avons pratiqué de nombreuses contaminations croisées; mais cette technique s'avère délicate car, selon les hôtes, le champignon manifeste des degrés d'endophytisme variable (Doustdar, 1958); chez la tomate, par exemple, on peut observer de simples tâches jaunes sans qu'il y ait apparition de fructifications caractéristiques. Nous sommes parvenus néanmoins à infecter le piment, la tomate, le concombre, l'artichaut et le cardon avec n'importe quel isolat. Diverses données bibliographiques confirment d'ailleurs cette non-spécificité du champignon; c'est ainsi que les isolats provenant de piment sont capables d'attaquer les genres Vicia et Hibiscus (Saad et al, 1972); les isolats provenant de tomate, les genres Cajanus, Cyamopsis, Vigna, Coriandrum (Pawar et al, 1985) et Olea (Ciccarone, 1955); les isolats provenant du concombre, les genres Vicia, Sesamum et Solanum (Saad et al, 1972); les isolats provenant de l'artichaut, le genre Solanum (Gaponenko, 1976). L taurica se distingue donc des autres oïdiums en faisant exception à la règle de stricte spécificité parasitaire. Notons, cependant, que des observations concernant le développement et la sporulation du champignon chez les différents hôtes mériteraient d'être réalisées, car le nombre élevé de plantes attaquées par un même isolat ne signifie pas forcément pléophagie mais, peut-être, grande diversité taxonomique (Rostam, 1983). D'ores et déjà, nous envisageons donc l'isolement de souches monospores.

Un dernier point retiendra notre attention, celui de la germination des spores en milieu liquide, propriété qui facilite beaucoup la mise en place de tests de screening de fongicides. Avec les genres Sphaerotheca et Erysiphe, la technique consiste à faire flotter les cotylédons sur la solution fongicide et à contaminer par soufflage à sec : dans ces conditions, on peut observer des sensibilités différentes aux fongicides en fonction du genre étudié (Caillol, 1988). Avec $L$ taurica, le test est très simplifié, mais il montre de manière évidente qu'aucune matière active, même à de très fortes concentrations, n'est capable de bloquer totalement la germination des spores; ce fait, auquel s'ajoute la capacité du champignon à se développer dans les tissus, explique sans doute les échecs rencontrés en lutte chimique (Piquemal et al 1985). 
Au sein des parasites obligatoires, $L$ taurica occupe donc une place très particulière, nécessitant, si l'on tient compte des difficultés actuelles de lutte, la poursuite des recherches.

\section{ANNEXE \\ Composition des différents milieux utilisés pour assurer, sur feuilles de tomate en survie, le développement de Leveillula taurica}

Milieu AIB. $\mathrm{pH}=6$

Macroéléments de Murashige et Skoog $100 \mathrm{ml} / \mathrm{I}$ Oligoéléments de Murashige et Shoog $1 \mathrm{ml} / \mathrm{l}$

Vitamines de Morel

Acide indol-butyrique (AIB)

Agar

Saccharose

$1 \mathrm{mg} / \mathrm{l}$

$1 \mathrm{mg} / \mathrm{l}$

$8 \mathrm{~g} / \mathrm{l}$

$5 \mathrm{~g} / \mathrm{l}$

Milieu KNOP. pH = 5,8

$\begin{array}{lr}\text { A } & 10 \mathrm{ml} / / \\ \text { B } & 10 \mathrm{ml} / / \\ \text { C } & 5 \mathrm{ml} / / \\ \text { D } & 5 \mathrm{ml} / / \\ \text { Oligoéléments } & 1 \mathrm{ml} / / \\ \text { Solution acide citrique et malique } & 1 \mathrm{ml} / 1 \\ \text { Gélose } & 20 \mathrm{~g} / 1 \\ \text { Saccharose } & 5 \mathrm{~g} / / \\ \text { Malt } & 1 \mathrm{~g} / / \\ \text { A : Nitrate de calcium } & 100 \mathrm{~g} / 1 \text { d'eau } \\ \quad \text { Nitrate de potasse } & 25 \mathrm{~g} / / \text { d'eau } \\ \text { B : Sulfate de magnésium } & 25 \mathrm{~g} / / \text { d'eau } \\ \text { C : Phosphate monopotassique } & 25 \mathrm{~g} / / \mathrm{l} \text { d'eau } \\ \text { D : Phosphate bipotassique } & 25 \mathrm{~g} / / \text { d'eau }\end{array}$

Milieu piment. $\mathrm{pH}=\mathbf{5 , 9}$

Macroéléments SNGN piment

Microéléments E $20 \mathrm{~A}$

Vitamines B 12

Méso-inositol

Pyridoxine $-\mathrm{HCl}$

Acide nicotinique

Thiamine - $\mathrm{HCl}$

Pantothénate de calcium

Biotine

Glycine

$\mathrm{Na}_{2}$ EDTA

$\mathrm{FeSO}_{4}, 7 \mathrm{H}_{2} \mathrm{O}$

2,4 D

$0,03 \mathrm{mg} / \mathrm{l}$

$50,30 \mathrm{mg} / 1$

$5,50 \mathrm{mg} / \mathrm{l}$

$0,70 \mathrm{mg} / \mathrm{l}$

$0,60 \mathrm{mg} / \mathrm{l}$

$0,50 \mathrm{mg} / \mathrm{l}$

$0,005 \mathrm{mg} / \mathrm{l}$

$0,100 \mathrm{mg} / \mathrm{l}$

$37,30 \mathrm{mg} / \mathrm{l}$

$27,80 \mathrm{mg} / 1$

$0,01 \mathrm{mg} / \mathrm{l}$
Kinétine

$0,01 \mathrm{mg} / \mathrm{l}$

Saccharose

$5 \mathrm{~g} / \mathrm{l}$

Agar

$10 \mathrm{~g} / \mathrm{l}$

Milieu MSE. pH $=6$

Macroéléments de Murashige et Skoog $100 \mathrm{ml} / \mathrm{I}$

Microéléments de Murashige et Skoog $1 \mathrm{ml} / \mathrm{l}$

Citrate ammoniacal de fer $2 \mathrm{ml} / \mathrm{l}$

Vitamines de Morel $2 \mathrm{ml} / \mathrm{l}$

Agar $8 \mathrm{~g} / \mathrm{l}$

Saccharose $\quad 5 \mathrm{~g} / \mathrm{l}$

Milieu RM. pH = 6

Macroéléments de Murashige et Skoog $100 \mathrm{ml} / \mathrm{l}$

Microéléments de Murashige et Skoog $1 \mathrm{ml} / \mathrm{I}$

Citrate ammoniacal de fer $2 \mathrm{ml} / \mathrm{l}$

Vitamines de Morel $2 \mathrm{ml} / \mathrm{I}$

Acide indol-butyrique $\quad 1 \mathrm{ml} / /$

Agar

$8 \mathrm{~g} / \mathrm{l}$

Saccharose

$5 \mathrm{~g} / \mathrm{l}$

\section{Milieu T51. pH 6}

Macroéléments de Murashige et Skoog $100 \mathrm{ml} / \mathrm{l}$

Microéléments de Heller $\quad 1 \mathrm{ml} / \mathrm{l}$

Vitamines de Morel $2 \mathrm{ml} / \mathrm{l}$

Agar $8 \mathrm{~g} / 1$

Saccharose $\quad 5 \mathrm{~g} / \mathrm{l}$

\section{Composition des solutions mères}

Macroéléments de Murashige et Skoog (mg/l)

$\mathrm{No}_{3} \mathrm{NH}_{4}$

$\mathrm{KNO}_{3}$

$\mathrm{Ca} \mathrm{Cl}_{2} 6 \mathrm{H}_{2} \mathrm{O} \quad 440$

$\mathrm{Mg} \mathrm{SO}_{4} 7 \mathrm{H}_{2} \mathrm{O}$

$\mathrm{KH}_{2} \mathrm{PO}_{4}$

$\mathrm{Na}_{2}$ EDTA

$\mathrm{Fe} \mathrm{SO}_{4} 7 \mathrm{H}_{2} \mathrm{O} \quad 27,8$

Microéléments de Murashige et Skoog (mg/l)

$\mathrm{HBO}_{3}$

6,2

$\mathrm{Zn} \mathrm{SO} 44 \mathrm{H}_{2} \mathrm{O}$

8,2

$\mathrm{Mn} \mathrm{SO}_{4} 4 \mathrm{H}_{2} \mathrm{O}$

22,6

IK

0,83

$\mathrm{Na}_{2} \mathrm{Mo} \mathrm{O}_{4} 2 \mathrm{H}_{2} \mathrm{O} \quad 0,35$

$\mathrm{SO}_{4} \mathrm{Cu} 5 \mathrm{H}_{2} \mathrm{O}$

0,025

$\mathrm{Co}_{2} 6 \mathrm{H}_{2} \mathrm{O}$ 
Vitamines de Morel (mg/l)

Calcium pantothénate

1

Méso-Inositol

Biotine

100

Acide nicotinique

Pyridoxine $\mathrm{H} \mathrm{Cl}$

Thiamine $\mathrm{H} \mathrm{Cl}$ tibilité sexuelle, sensibilité aux fongicides. Mémoire ENITA Bordeaux

Ciccarone A (1955) Indizi di specializzazione del parasitismo in Leveillula taurica (Lev) Arn. Not Mal Piante 29, 165-169

Daubeze AM, Pochard E, Palloix A (1989) Inheritance of resistance to Leveillula taurica and relation to other phenotypic characters in the haplodiploid progeny issued from an African pepper line. Eucarpia 7th meeting on capsicum and eggplant. 27-30 juin, Kragujevac Yugoslavia, 229-232

Diop-Bruckler M (1989) Développement de Leveillula taurica en fonction des facteurs climatiques et sensibilité de Capsicum annuum à différents stades végétatifs. J Phytopathol 126, 104-114

Doustdar EM (1958) Les Érysiphacées de I'Iran. Thèse de Phytopath Fac Sci, Paris

Fauvel C, Molot PM (1985) Une enceinte de conception fort simple pour l'étude des Oïdiums. Cah Techn INRA 11, 9-20

Gaponenko NI (1976) Specialization of fungi of the genus Leveillula Arn. Mikol Fitopatol 10, 293-297

Microéléments E 20 A (mg/l)

$\begin{array}{lr}\mathrm{MnSO}_{4}, \mathrm{H}_{2} \mathrm{O} & 11,065 \\ \mathrm{ZnSO}_{4}, 7 \mathrm{H}_{2} \mathrm{O} & 1,812 \\ \mathrm{H}_{3} \mathrm{Bo}_{3} & 1,575 \\ \mathrm{KI} & 0,345 \\ \mathrm{NaMoO}_{4}, 2 \mathrm{H}_{2} \mathrm{O} & 0,094 \\ \mathrm{CuSO}_{4}, 5 \mathrm{H}_{2} \mathrm{O} & 0,008 \\ \mathrm{CoCl}_{2}, 6 \mathrm{H}_{2} \mathrm{O} & 0,008\end{array}$

\section{Microéléments de Heller (mg/l)}

\begin{tabular}{|c|c|}
\hline $\mathrm{H}_{3} \mathrm{Bo}_{3}$ & $\begin{array}{l}1 \\
1\end{array}$ \\
\hline $\begin{array}{l}\mathrm{Zn} \mathrm{SO} \mathrm{SO}_{4} 7 \mathrm{H}_{2} \mathrm{O} \\
\mathrm{Mn} \mathrm{SO}_{4} 1 \mathrm{H}_{2} \mathrm{O}\end{array}$ & $\begin{array}{l}1 \\
0,076\end{array}$ \\
\hline IK & 0,01 \\
\hline $\mathrm{SO}_{4} \mathrm{Cu} 5 \mathrm{H}_{2} \mathrm{O}$ & 0,03 \\
\hline $\mathrm{Al} \mathrm{Cl}_{3} 6 \mathrm{H}_{2} \mathrm{O}$ & 0,05 \\
\hline $\mathrm{Ni} \mathrm{Cl}_{2} 6 \mathrm{H}_{2} \mathrm{O}$ & 0,03 \\
\hline
\end{tabular}

\section{RÉFÉRENCES}

Besri M, Hormatallah A (1985) Manifestation et mode de conservation de Leveillula taurica, agent de l'oïdium de la tomate au Maroc. Phytopathol Z 112, 348-354

Blancard D, Rieuf P (1981) La recrudescence d'un oïdium des cultures maraîchères dans le Midi de la France. Phytoma 333, 43-44

Caillol A (1988) Étude du comportement d'isolats d'oïdium des Cucurbitacées : virulence sur gamme d'espèces, virulence sur variétés de melon, compa-
Homma $\mathrm{Y}$, Arimoyo $\mathrm{Y}$, Takahashi $\mathrm{H}$, Ishikawa I, Matsuda I, Misato T (1980) Studies on pepper powdery mildew. I. Conidial germination, hyphal elongation and hyphal penetration on pepper leaf. Ann Phytopathol Soc Jpn 46, 140-149

Koren A (1978) Aspects of controlling Leveillula taurica (Lèv) Arn in canning tomatoes. Thesis. Hebrew University, Jerusalem

Kunoh H, Kohno M, Tashiro S, Ishizaki H (1979) Studies of the powdery mildew fungus, Leveillula tauri$c a$, on green pepper. II. Light and electron microscopic observation of the infection process. Canad $J$ Bot 57, 2501-2508

Molot PM, Lecoq H (1986) Les oïdiums des Cucurbitacées. I. Données bibliographiques. Travaux préliminaires. Agronomie 6 (4), 355-362

Molot PM, Leroux JP, Ferrière H (1987a) Les oïdiums des cucurbitacées. II. Mise au point d'une technique de conservation des souches en culture axénique. Agronomie 7, 339-343

Molot PM, Leroux JP, Ferrière H (1987b) Mise au point d'une technique de conservation en conditions axéniques de différents oïdiums sur feuilles adultes racinées. SFP $32^{e}$ Colloque, 14-15 mai, Angers

Palti J (1971) Biological characteristics, distribution thol mediterr 10, 139-153

Palti J (1988) The Leveillula mildews. In : "The Botanical Review" 54, 423-535

Pawar RN, Utikar PG, Shinde PA (1985) A study of powdery mildew of chilli. J Maharashtra Agric Univ 10,218

Piquemal JP, Blancard D, Diop-Bruckler M, GebreSelassie K, Lecoq H, Lot H, Michel MJ (1985) Quelques problèmes d'actualité en cultures légumières. and control of Leveillula taurica (Lèv). Arn Phytopa- 
In : «Premières journées des Maladies des plantes", ANPP, Paris, 479-480

Pochard E (1982) Rapport d'activités 1981-1982 de la Station d'amélioration des plantes maraîchères. INRA, Montfavet

Reuveni R, Rotem J (1973) Epidemics of Leveillula taurica on tomatoes and peppers as affected by conditions of humidity. Phytopathol Z 76, 153-157

Rostam S (1983) Biologie, écologie, systématique de quelques Leveillula. Thèse de phytopathologie, Faculté des sciences de Toulouse
Saad AT, Abul-Hayja Z, Sonmez MM (1972) Investigations on Leveillula species in Lebanon. Proc //le Congr Mediterr Phytopathol Union, 147-154

Tramier R (1963) Étude préliminaire du Leveillula taurica (Lev) Arn dans le Midi de la France. Ann Epiphyt 14, 355-369

Zaracovitis C (1964) Factors in testing fungicides against powdery mildews. The germination of the conidia in vitro. Ann Inst Phytopathol Benaki NS 6, 73-106 